University of Warwick institutional repository: http://go.warwick.ac.uk/wrap This paper is made available online in accordance with publisher policies. Please scroll down to view the document itself. Please refer to the repository record for this item and our policy information available from the repository home page for further information.

To see the final version of this paper please visit the publisher's website. Access to the published version may require a subscription.

Author(s): Garima KESHRI, Mike CHALLEN, Tim ELLIOTT and Naresh MAGAN

Article Title: Differentiation of Agaricus species and other homobasidiomycetes based on volatile production patterns using an electronic nose system Year of publication: 2003 Link to published version: http://dx.doi.org/10.1017/S0953756203007743 Publisher statement: None 


\title{
Differentiation of Agaricus species and other homobasidiomycetes based on volatile production patterns using an electronic nose system
}

\author{
Garima KESHRI $^{1}$, Mike CHALLEN ${ }^{2}$, Tim ELLIOTT ${ }^{2}$ and Naresh MAGAN ${ }^{1 *}$ \\ ${ }^{1}$ Applied Mycology Group, Biotechnology Centre, Cranfield University, Silsoe, Bedford MK45 4DT, UK. \\ ${ }^{2}$ Horticulture Research International, Wellesbourne, Warwick CV35 9EF, UK. \\ E-mail:n.magan@cranfield.ac.uk
}

Received 18 May 2002; accepted 20 March 2003.

\begin{abstract}
Comparisons of the qualitative volatile production patterns between seven species of Agaricus, and between two of Volvariella and Pleurotus and one Coprinus species when grown at $25{ }^{\circ} \mathrm{C}$ on agar media for $14 \mathrm{~d}$ were made. There was good reproducibility between the volatile production patterns of the same species using an electronic nose unit with a 14 conducting sensor polymer array. Principle Component Analysis (PCA) showed that it was possible to discriminate between five of the seven Agaricus species, but that some overlap occurred between the others. Cluster analysis showed that there was also overlap between some species with the tropical collection of $A$. bitorquis separating out from the others. The volatile production profile of the commercial A. bisporus was close to that of a wild species, A. campestris. A. bisporus could be readily differentiated from other non-Agaricus species. This study demonstrates the potential for using electronic nose systems to rapidly differentiate mycelial cultures of homobasidiomycete mushrooms.
\end{abstract}

\section{INTRODUCTION}

There has recently been interest in rapid methods for the differentiation between related fungal species using a range of techniques including molecular methods, isoenzyme profiles, hydrolytic production profiles, and more recently volatile production patterns (Schnurer, Olsson \& Borjesson 1999, Magan \& Evans 2000).

Despite the importance of odour in the identification of homobasidiomycete mushrooms, no attempt has been made to detect and differentiate volatile production patterns of closely related species. Indeed Cappelli (1984) suggested that the smell of one species may vary according to the weather. Often it is necessary to bruise or crush the flesh in order to detect such odours. Table 1 shows the descriptors currently used to distinguish different homobasidiomycetes (Cappelli 1984, Jordan 1995). Electronic nose (e-nose) systems could provide a more definitive, less subjective method to define mushroom odours and studies like those described here could be used to provide benchmark odour values for a range of homobasidiomycetes, especially mycelial cultures, including Agaricus, to support taxonomic definitions.

\footnotetext{
* Corresponding author.
}

Recent studies with spoilage fungi suggest the possibility of early detection of volatile production patterns using odour mapping devices which could enable detection of moulds contaminating food raw materials (Schnurer, Olsson \& Borjesson 1999, Magan \& Evans 2000). Indeed, developments in this technology suggest potential for rapid differentiation between species based on qualitative volatile production patterns. For example, Keshri et al. $(1998,2001)$ showed that spoilage fungi could be differentiated within $48 \mathrm{~h}$ of spore germination, both in agar media and in food matrices; Gibson et al. (1997) could distinguish between bacteria, and Magan, Pavlou \& Chrysanthakis (2001) between spoilage bacteria and yeasts in milk media. To our knowledge no studies have been carried out to try and differentiate fresh mushrooms or mycelial cultures, although some freeze dried cultures have been examined (Breheret et al. 1995). Mushroom sporophores are more odiferous than mycelial cultures, and if the technology can discriminate between such mycelia, then it should prove even more effective using the mushrooms themselves.

The objective of this study was to evaluate the potential of an e-nose system for the differentiation of homobasidiomycete mushroom species based on mycelial cultures. In this paper we describe the qualitative patterns of volatile production for seven different species 
Table 1. Homobasidiomycete mushroom species used in electronic nose comparison; authorities, classification and description of odours.

\begin{tabular}{lll}
\hline Species & Classification $^{\mathrm{a}}$ & Odour descriptions $^{\mathrm{b}}$ \\
\hline Agaricus arvensis & Agaricaceae, Flavescentes sect. Arvenses & Smell of almonds, strong of aniseed \\
A. bisporus & Agaricaceae, Rubescentes sect. Bitorques & Smell pleasant, marked, strong of mushroom \\
A. bitorquis & Agaricaceae, Rubescentes sect. Bitorques & Smell marked, agreeable, not distinctive \\
A. campestris & Agaricaceae, Rubescentes sect. Agaricus & Smell agreeable, not of almonds \\
A. nivescens & Agaricaceae, Flavescentes sect. Arvenses & Smell of almonds \\
A. maleolens & Agaricaceae, Rubescentes sect. Bitorques & Slightly fishy \\
A. subfloccosus & Agaricaceae, Rubescentes sect. Sanguinolenti & Smell somewhat unpleasant \\
Coprinus cinereus & Coprinaceae, Laatuli sect. Lagopus & Not distinctive \\
Pleurotus ostreatus & Lentinaceae & Not distinctive \\
P. sajor-caju & Lentinaceae & No description \\
Volvariella bombycina & Pluteaceae & Strong, pleasantly fungoid \\
V. volvacea & Pluteaceae & No description \\
\hline
\end{tabular}

a Sections in the genus Agaricus based on Cappelli (1984).

b From Cappelli (1984) and (or) Jordan (1995).

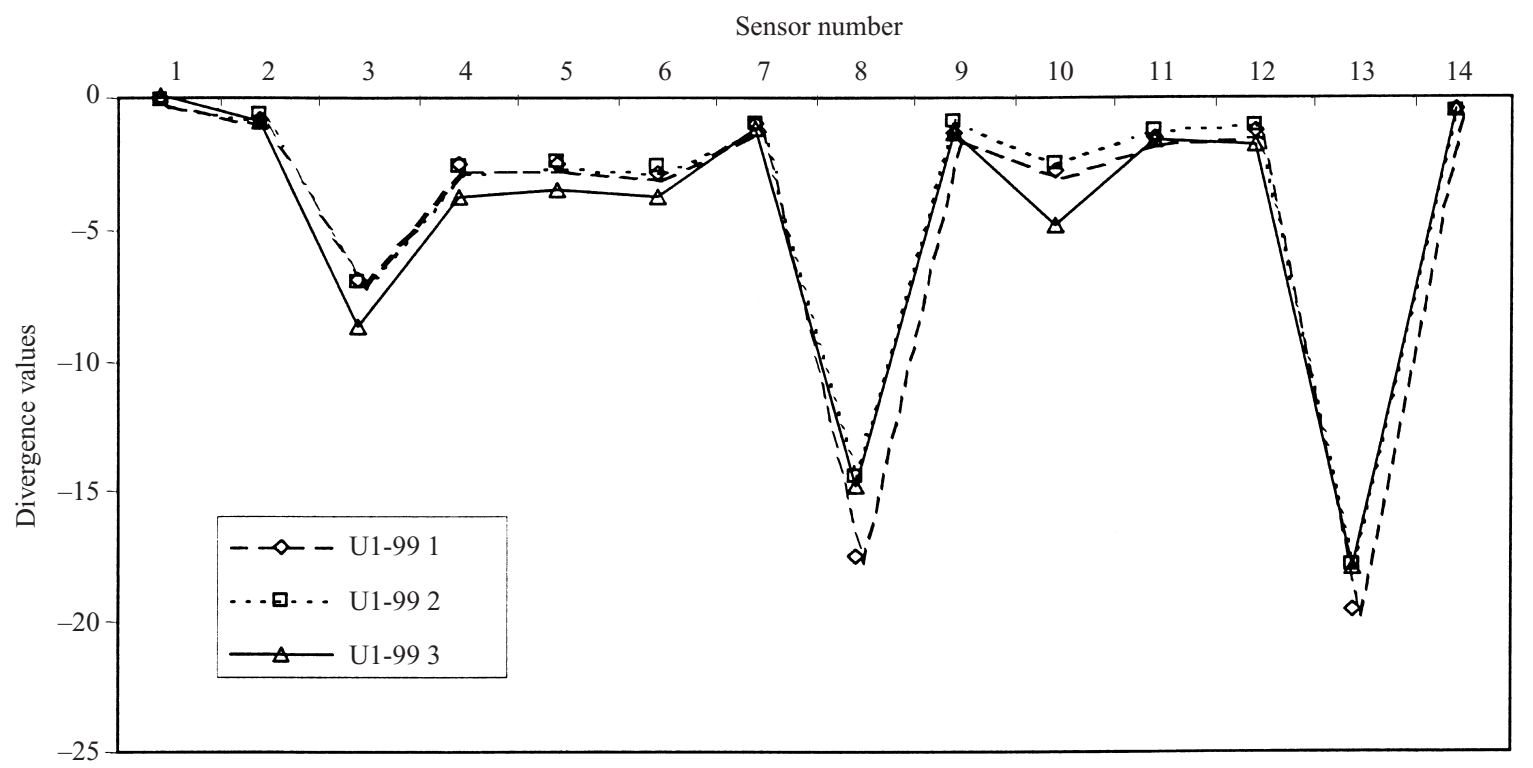

Fig. 1. Comparison of divergence response of 14 sensor conducting polymer array for randomised replicates of mycelial cultures of Agaricus bisporus (U1-99) after $14 \mathrm{~d}$ incubation at $25^{\circ} \mathrm{C}$.

of Agaricus, and for four other species from the diverse genera Volvariella, Pleurotus and Coprinus.

\section{MATERIALS AND METHODS}

\section{Fungal species used in this study}

Species within Agaricus are separated into two large groups, Rubescentes and Flavescentes, based on macrochemical reactions to the Schaffer colour assay. Within these, there are numerous sections and species (Cappelli 1984). In this study we have used collections that span both large groups and fall within four of the sections defined by Cappelli (1984).

The Agaricus species were: Agaricus bisporus U1-99, a wild strain W7 originally identified as A. macrosporus (Elliott 1978) but recently reclassified as A. maleoleons (Challen \& Noble, unpubl.), A. nivescens W6I (CalvoBado et al. 2000), A. arvensis 93-7 (Calvo-Bado et al.
2000), A. campestris W1S, A. bitorquis W20 (Pahil, Smith \& Elliott 1991) and A. subfloccosus W4II (Smith \& Love 1995). The other species examined were $\mathrm{Vol}$ variella bombycina R83 (Elliott \& Challen 1985), V. volvacea R171, Pleurotus sajor-caju R90, Pleurotus ostreatus R155 and Coprinus cinereus $\mathrm{CcH} 9$ (Challen et al. 1994). All strains were recovered from and are preserved in the HRI liquid nitrogen culture collection (Challen \& Elliott 1986).

Mycelial cultures were grown up on malt peptone medium (malt extract, Oxoid L39 $20 \mathrm{~g}^{-1}$, mycological peptone, Oxoid L40, $5 \mathrm{~g}^{-1}$ ) and $1.2 \% \mathrm{wt} \mathrm{vol}^{-1}$ agar (Oxoid Technical No. 3, L13). The agar medium was poured into $9 \mathrm{~cm}$ Petri plates and they were inoculated in three places on each plate with $4 \mathrm{~mm}$ diam agar plugs taken with a cork borer from the growing margin of colonies on the same medium for $14 \mathrm{~d}$. Four replicate plates of each species were incubated at $25{ }^{\circ} \mathrm{C}$ for 14-21 d prior to e-nose measurements being carried out. All experiments were repeated once. 


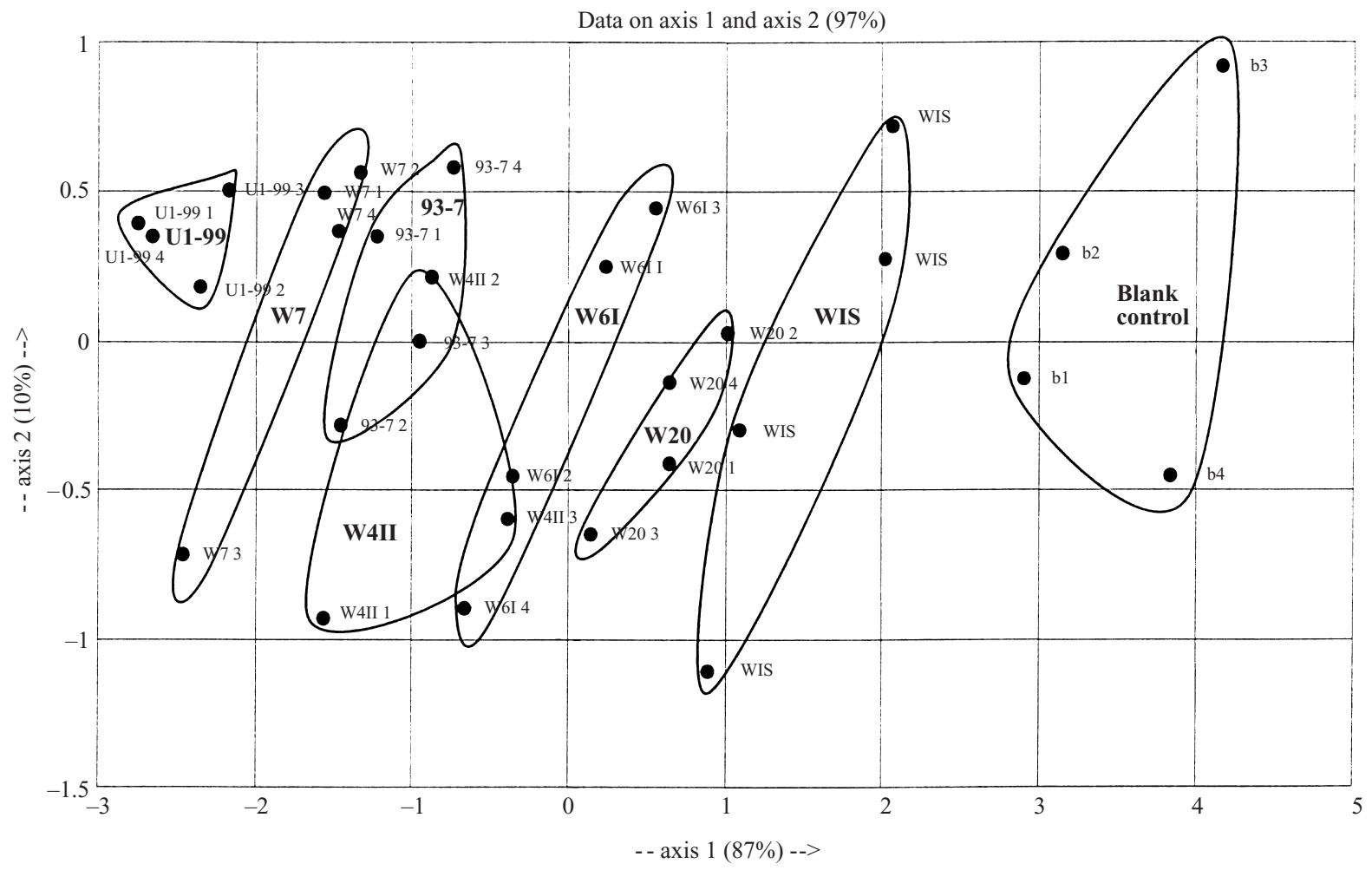

Fig. 2. Principle component analysis (PCA1, PCA2) of the data for all Agaricus species after $14 \mathrm{~d}$ incubation showing the groupings for the control blanks and all the species. PCA based on divergence data of all 14 sensors. Key to species: U1-99, $A$. bisporus; W7, A. maleolens; 93-7, A. arvensis; W4II, A. subfloccosus; W6I, A. nivescens; W20, A. bitorquis; W1S, A. campestris.

\section{Sampling with electronic nose and data analyses}

Individual plates were placed in a sampling bag $(500 \mathrm{ml})$ and filled with filter sterilised air and sealed (Keshri \& Magan 2001). After $2 \mathrm{~h}$ equilibration at 25 , the headspace of each bag was sampled in a randomised fashion using a Bloodhound BH114 e-nose unit (Bloodhound Sensors, Leeds). This system uses 14 conducting polymer sensors which work best under ambient conditions. To minimise the influence of relative humidity all samples were prefiltered prior to entering the e-nose unit. The interaction of volatile compounds and the conducting polymer surface produces a change in resistance which can be amplified and analysed through the e-nose software package system. The flow rate over the sensors was fixed at $4 \mathrm{ml} \mathrm{min}{ }^{-1}$ to generate the sensor baselines. Four sensor parameters can be studied with this system: adsorption (maximum rate of change of resistance), desorption (maximum negative rate of change of resistance), divergence (maximum step response) and area (area under the actual sensor curve). The divergence response was primarily used for this study, and the sampling profile was set at $15 \mathrm{~s}$ of adsorption and $22 \mathrm{~s}$ of desorption. This has been detailed elsewhere (Pavlou et al. 2000).

Normalised e-nose data were analysed using the programme xlStat (Microsoft Excel add-in program). Principle Component Analysis (PCA) and Cluster Analysis (CA) techniques were applied to differentiate and classify the fungal species. To carry out analyses the response from all the sensors were used. Cluster analysis was based on the Malahanobi's squared distance between groups obtained in the discriminant analysis at the $95 \%$ confidence limit.

\section{RESULTS AND DISCUSSION}

Fig. 1 shows the divergence data for the volatile profiles for three replicates of a single treatment (Agaricus bisporus U1-99) for all 14 sensors. The between replicate standard error was small, with an overall average of $<10 \%$. The PCA (axis 1 and 2) accounts for $97 \%$ of the data for the Agaricus species and indicates the groupings obtained based on the volatile production profiles (Fig. 2). This also shows that there was differentiation between the uninoculated blank medium alone (control), and the A. bisporus (U1-99) strain at the two ends of the spectrum. Overall, PCA differentiation between the Agaricus species was robust, except for $A$. subfloccus (W4II) which overlapped with A. arvensis (93-7) and A. nivescens (W6I).

Examination of the data using Cluster Analysis (CA, Fig. 3) revealed that there were 3 different groups. A. bisporus (U1-99) and A. campestris (W1S) were grouped together, A. bitorquis (W20) was completely separated out, and the remaining species clustered from a third branch. Within the large cluster, two subgroups comprised A. nivescens (W6I) with $A$. subfloccosus (W4II) and A. maleolens (W7) with the control. Interestingly $A$. avensis (93-7) was differentiated from $A$. nivescens (W6I). These two species are inter-related 


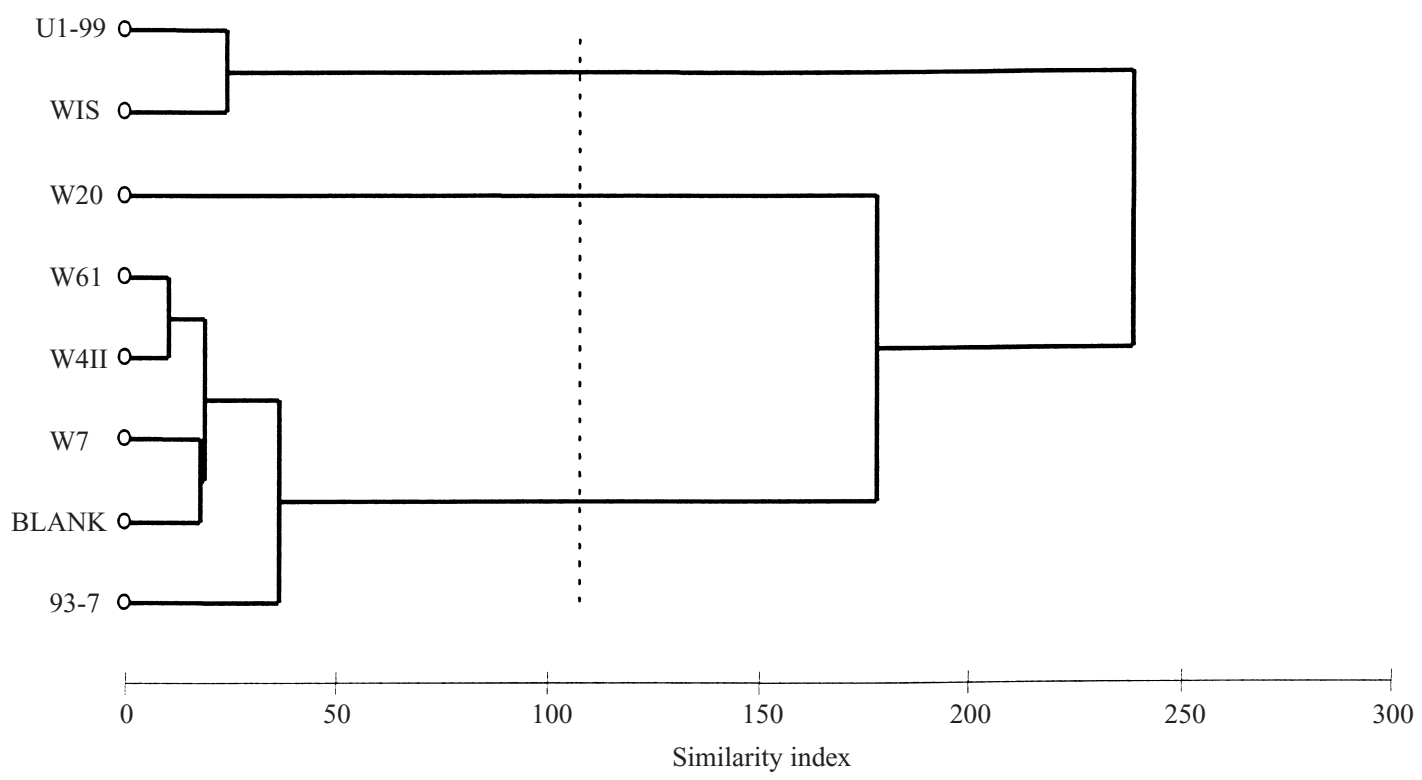

Fig. 3. Cluster analysis $(P=0.05)$ of the data showing discrimination between the different species of Agaricus. The index indicates the relative distance between treatments. Key to species: see Fig. 1.

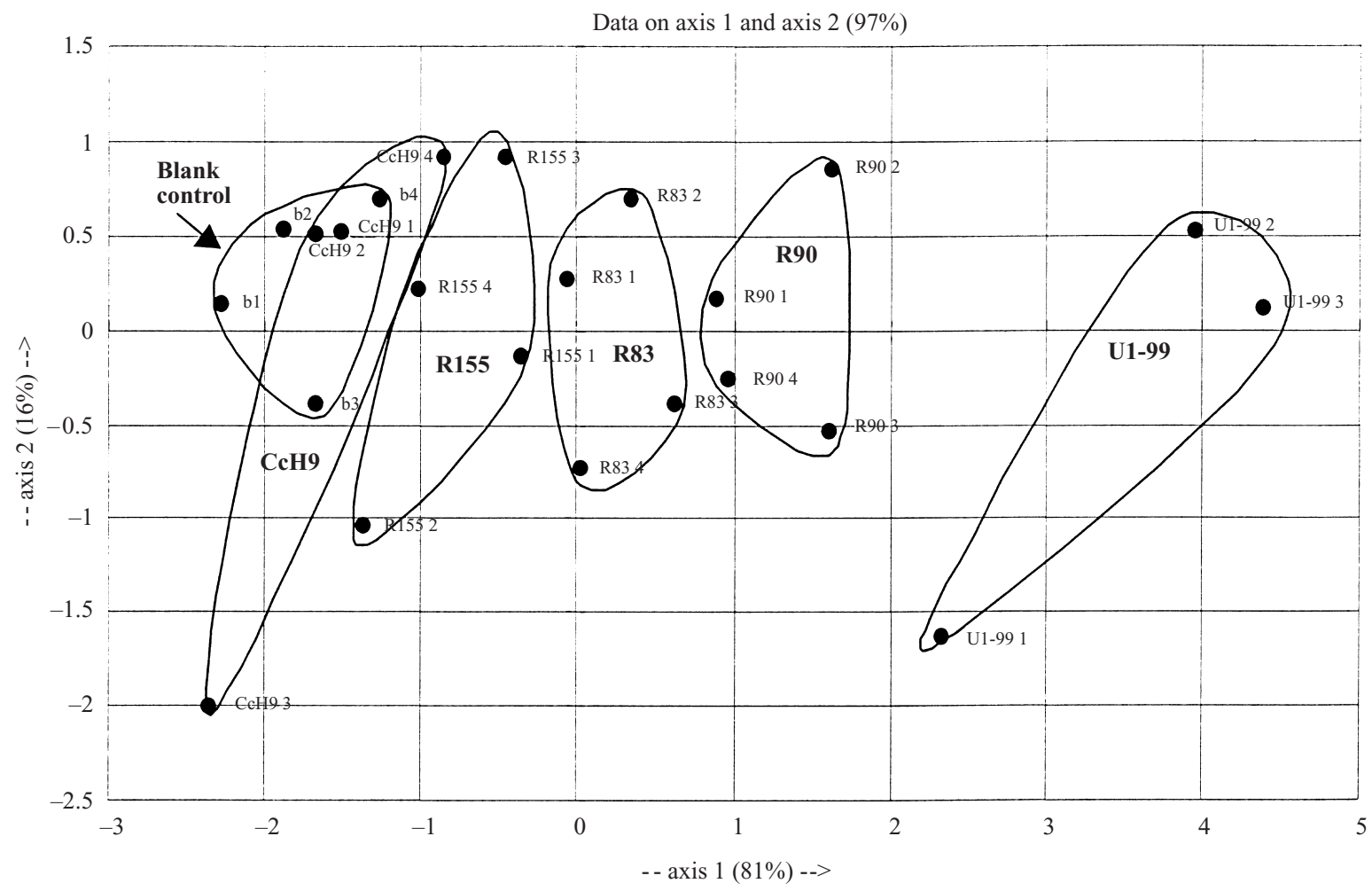

Fig. 4. Principle component analysis (PCA1, PCA2) of the divergence data of the 14 conducting polymer sensor array for comparisons between the genera Agaricus, Pleurotus, Coprinus and Volvariella after $14 \mathrm{~d}$ incubation. All treatments and replicates randomly sampled. Key to species: U1-99, A. bisporus; CcH9, C. cinereus; R155, P. ostreatus; R83, V. bombycina; R90, P. sajor-caju.

but can be distinguished by morphological, compatibility and DNA analyses (Calvo-Bado et al. 2000). Although some minor overlapping occurred between samples within the Agaricus genus, the differentiation between diverse homobasidiomycete genera was more obvious based on volatile production patterns. This suggests that e-nose may need further optimisation to detect subtle variations in volatile production patterns in species within a single genus.

Fig. 4 shows that when comparing the data for A. bisporus (U1-99) and species from other homobasidiomycete genera the PCA (axis 1 and 2) accounts 
for $97 \%$ of the data. This shows that mycelial cultures of A. bisporus (U1-99) could be differentiated from Volvariella, Pleurotus and Coprinus species. There was very low volatile production by $C$. cinereus this resulted in an overlap with the blank control sample.

This study suggests that discriminating between genera may be readily achieved on the basis of volatile production profiles of mycelial cultures and shows that within Agaricus even some closely related species can be differentiated. It has been established that growth media and culture age affects type and concentration of volatiles produced (Schnurer, Olsson \& Borjesson 1999, Magan \& Evans 2000). Thus, better discrimination may be possible by optimising these criteria. There is only one reported investigation of an attempt to use e-nose systems to assess differences in mushroom odours. Freeze dried cultures of 9 wild species were examined with a laboratory e-nose system and GC. Both techniques differentiated the species into the same four groups. One of the mushroom species was clearly discriminated from the others (Breheret et al. 1995). This work was unfortunately not followed up with any studies on actively growing colonies or basidiomes.

Mushroom identification is still largely based on gross morphology. Recently molecular techniques such as the sequencing of rRNA genes are becoming increasingly pervasive in the resolution of fungal taxonomic groupings (Hibbett et al. 1997, Monocalvo et al. 2000). In Agaricus, ITS sequences have been used to differentiate species that are otherwise difficult to distinguish (CalvoBado et al. 2000). It is therefore appropriate to compare molecular and volatile profiling technologies with respect to their ability to discriminate species. In this study, the CA cluster groups were not always consistent with relationships determined from the molecular analysis. In terms of genetic identity, $A$. arvensis and $A$. nivescens are closely related, both fall within the section Arvenses and although different species, they are capable of limited interbreeding (Calvo-Bado et al. 2000). A more striking difference is the comparison between $A$. bisporus and $A$. subfloccosus. ITS sequencing and other genetic analysis indicate that these two species are very closely related (Challen, Kerrigan \& Callac, unpubl.) while in this study, A. bisporus and A. subfloccosus fall into different CA clusters. Odour cluster groups can therefore differ from those determined by ITS sequencing and, our study shows that a multi-array e-nose can prove more discriminatory than molecular markers limited to short specific sequences. E-nose technology is a fast moving field and there are continuing improvements in both the sensitivity of the sensor arrays and the range of detectable volatiles. Improved discrimination between species is highly probable and this opens the possibility for database development to assist with rapid identification of such fungal species.

\section{REFERENCES}

Breheret, S., Talou, T., Bourroaunet, B. \& Gaset, A. (1995) On line differentiation of mushroom aromas by combined head space/ multi-odour gas sensor devices. In Proceedings of Bioflavour 5, (P. Etievant \& P. Schreier, eds): 103-107. INRA, Dijon.

Calvo-Bado, L., Noble, R., Challen, M., Dobrovin-Pennington, A. \& Elliott, T. (2000) Sexuality and genetic identity in the Agaricus section Arvenses. Applied and Environmental Microbiology 66: 728-734.

Cappelli, A. (1984) Agaricus L.: Fr. (Psalliota Fr.). Libreria Editrice Biella Giovanna, Sorano, Italy.

Challen, M. P. \& Elliott, T. J. (1986) Polypropylene straw ampoules for the storage of microorganisms in liquid nitrogen. Journal of Microbiological Methods 5: 11-23.

Challen, M. P., Bhattiprolu, G. R., Warner, P. J. \& Elliott, T. J. (1994) Cloning the Coprinus bilanatus TRP2 gene and its use as a selectable marker in transformation. Mycological Research 98: 179-185.

Elliott, T. J. (1978) Comparative sexuality in Agaricus species. Journal of General Microbiology 107: 113-122.

Elliott, T. J. \& Challen, M. P. (1985) The breeding system of the silver-silk straw mushroom, Volvariella bombycina. Mushroom Newsletter for the Tropics 6: 3-8.

Gibson, T. D., Prosser, O., Hulbert, J. N., Marshall, R. W., Cocoranm, P., Lowery, E. E. \& Ruck-Keene, E. A. (1997) Detection and simultaneous identification of microorganisms from head space samples using an electronic nose. Sensors and Actuators B 44: 413-422.

Hibbett, D. S., Pine, E. M., Langer, E., Langer, G. \& Donoghue, M. J. (1997) Evolution of gilled mushrooms and puffballs inferred from ribosomal DNA sequences. Proceedings of National Academy of Sciences, USA 94: 12002-12006.

Jordan, M. (1995) The Encyclopaedia of Fungi of Britain and Europe. David \& Charles, Newton Abbot.

Keshri, G., Magan, N. \& Voysey, P. (1998) Use of an electronic nose for the early detection and differentiation between spoilage fungi. Letters in Applied Microbiology 27: 261-264.

Keshri, G. \& Magan, N. (2001) Detection and differentiation between mycotoxigenic and non-mycotoxigenic strains of two Fusarium spp. using volatile production profiles and hydrolytic enzymes. Journal of Applied Microbiology 89: 825-833.

Magan, N. \& Evans, P. (2000) Volatiles and indicators of fungal activity and differentiation between species, and the potential use of electronic nose technology for early detection of grain spoilage. Journal of Stored Product Research 36: 319-340.

Magan, N., Pavlou, A. \& Chrysanthakis, I. (2001) Milk-sense: a volatile sensing system recognises spoilage bacteria and yeasts in milk. Sensors and Actuators B 72: 28-34.

Monocalvo, J.-M., Lutzoni, F. M., Rehner, S. A., Johnson, J. \& Vilgalys, R. (2000) Phylogenetic relationship of agaric fungi based on nuclear large subunit ribosomal DNA sequences. Systematic Biology 49: 278-305.

Pahil, V.S., Smith, J. F. \& Elliott, T. J. (1991) The testing and improvement of high temperature, wild Agaricus strains for use in tropical and sub-tropical climates. Mushroom Science 13: 589-599.

Pavlou, A., Magan, N., Sharp, D., Brown, J., Barr, H. \& Turner, A. P. F. (2000) An in vitro rapid odour detection and recognition model in discrimination of $H$. pylori and other gastroeosophageal pathogens. Biosensors and Bioelectronics 15: 333-342.

Schnurer, J., Olsson, J. \& Borjesson, T. (1999) Fungal volatiles as indicators of food and feed spoilage. Fungal Genetics and Biology 27: 209-217.

Smith, J. F. \& Love, M. E. (1995) Investigations into the cultural requirements of a brown capped Agaricus strain (W4 II) isolated from Cupressus leaf litter. Journal of Horticultural Science 70: 963-974.

Corresponding Editor: J. I. Lelley 